\title{
A case of complicated bullous systemic lupus erythematosus managed with
}

\section{Rituximab}

Ananda Mohan Chakraborty, Srabani Ghosh, Soumitra Ghosh, Abhishek Chowdhury, and Debashish Mandal

Institute of Post Graduate Medical Education and Research, Kolkata, India

\section{CASE STUDY}

Please cite this paper as: Chakraborty A, Ghosh S, Ghosh S, Chowdhury A, Mondal D. A case of complicated bullous systemic lupus erythematosus managed with Rituximab. AMJ 2017;10(12):993-996.

https://doi.org/10.21767/AMJ.2017.3197

\section{Corresponding Author:}

Ananda Mohan Chakraborty

Department of General Medicine

IPGME\&R and SSKM Hospital

244 AJC Bose Road, Kolkata-700020. West Bengal, India

Email: anandamohanchakraborty@gmail.com

\section{ABSTRACT}

Skin Involvement is seen in nearly 76 per cent of Systemic lupus erythematosus (SLE) patients, but bullous lesion however accounts for less than one per cent of cutaneous manifestation of lupus erythematosus. A 21-year-old female presented with fever, polyarthalgia and vesicobullous skin lesion and acute renal failure. Clinico-pathological evaluation imparted that she was having bullous systemic lupus erythematosus (BSLE) with lupus nephritis. She was put on rituximab after initial resuscitation. She responded dramatically with resolution of skin lesion and recovery from acute renal failure. She was followed up for one year without complication with stable disease course.

\section{Key Words}

BSLE, Rituximab, Vesico-bullous lesion, Lupus nephritis

\section{Implications for Practice:}

\section{What is known about this subject?}

Bullous Systemic Lupus Erythematosus has been reported very infrequently in literature. It is a skin manifestation of SLE.

\section{What new information is offered in this case study?}

Response to Rituximab a CD20 monoclonal antibody has not been reported adequately in literature which has been shown here.

3. What are the implications for research, policy, or practice?

Patient with SLE with extensive bullous lesion can be managed with Rituximab as an alternative to Dapsone. SLE nephropathy too can be controlled by Rituximab therapy.

\section{Background}

Systemic lupus erythematosus (SLE) is a multisystem autoimmune disease. It can virtually involve all organ systems. Skin Involvement is seen in nearly 76 per cent of all lupus patients, ${ }^{1}$ most common are malar rash, discoid rash and photo sensitivity. Bullous lesion, however accounts for less than one per cent of cutaneous manifestation of lupus.1 Bullous systemic lupus erythematosus (BSLE) is a distinctive entity characterised by histological appearance of sub epidermal bulla with deposition of pan immuno reactants and circulating antibody against type VII collagen (NC1 domain) and occasionally other antigens like laminin 5, laminin 6, and bullous pemphigoid antigen (BP230). ${ }^{2}$ BSLE frequently parallels internal organ involvement. 3 In this case report we have reported a case of bullous systemic lupus erythematosus (BSLE) who presented with an organ threatened condition in the form of rapidly progressive glomerulonephritis (RPGN).

\section{Case details}

A 21-year-old, newly married female without any living issue and foetal loss, resident of eastern India presented to IPGME\&R hospital with presenting complaints of low to moderate grade intermittent fever (with occasional high rise of temperature) for last six months, pain, redness and swelling of both large and small joints (mostly additive) for last six months. Also rapidly progressive painful vesicobullous skin lesion involving both sun exposed and nonexposed areas i.e. face, neck, upper back, limbs and 
abdomen for last one month in multiple crops, with rupture of vesicles and draining of clear to turbid and occasionally haemorrhagic fluid. Some of the lesions subsided with scar formation. She also had history of scanty micturition for last 15 days with swelling of limbs and face for last 10 days.

Clinical examination revealed poor nutritional status with moderate pallor, bilateral pitting pedal oedema with facial puffiness, elevated jugular venous pressure (JVP) and raised temperature $\left(101^{\circ} \mathrm{F}\right)$. Her pulse rate was $116 / \mathrm{min}$, Blood pressure was $160 / 70 \mathrm{~mm}$ of $\mathrm{Hg}$ and respiration rate of $38 / \min$.

Examination of skin revealed extensive vesicles and bullas with partial denudation in limbs, face, neck and in trunk. Nikolsky's sign and bulla spread sign was positive. There was evidence of secondary infection and scarring (Figures 1-5).

Apart from inflammatory polyarthritis and left sided pleural effusion and mild hepato splenomegaly other systems were within normal limit.

Laboratory Examination shows:

a) Blood Biochemistry and haematology: (Table 1),

b) Chest X-RAY: Left more than right sided mild pleural effusion.

c) Echocardiography: Mild Pericardial Effusion $10 \mathrm{cc}$, EF $65 \%$, No features of Myocarditis.

d) USG: Liver $15.9 \mathrm{~cm}$, normal shape and echotexture. Mild Splenomegaly with mild ascites. Kidney Right $11.8 \mathrm{~cm}$, left $11 \mathrm{~cm}, \mathrm{CMD}$ accentuated and bilateral cortical echogenicity increased, suggestive of renal parenchymal disease.

e) Urine Routine: Proteinuria 3+, RBC 4-6/ HPF, Pus cell 1-2/HPF.

f) Creatinine clearance: $20 \mathrm{ml} / \mathrm{min}$.

g) 24 hour urinary protein estimation: $4.25 \mathrm{gm}$ in 24 hour. (Volume 1.45lit)

h) Ascitic fluid study: Exudative with lymphocyte predominance.

i) Pleural fluid study: Exudative, Low ADA, lymphocyte predominance.

j) Serum ANA: ANA positive with 4+ homogeneous patterns with cytoplasmic position in 1:160 dilutions.

k) ANA Profile: anti ds DNA 2+, anti-Ribosomal P protein $3+$, anti-Histone + , anti-Smith + , anti SS-A +.

I) C3 and C4: $28 \mathrm{mg} / \mathrm{dl}$ and $6.24 \mathrm{mg} / \mathrm{dl}$ respectively.

m) hS CRP: $<0.03 \mathrm{mg} / \mathrm{dl}$. Ferritin: $771 \mu \mathrm{gm} / \mathrm{L}$.

n) Thyroid and other hormonal profile were unremarkable. o) Skin Biopsy: Sub epidermal separation with neutrophilic infiltration in papillary dermis. Linear staining of the basement membrane zone with IgG, $\operatorname{IgM}, \operatorname{IgA}, \quad \mathrm{C} 3$, and fibrinogen in direct immunoflurescence microscopy. Split Band is seen in the dermal side of the split (Floor pattern) with these Immuno reactants. Suggestive of Bullous lesion. Indirect immunoflurescence showed antibody to type VII collagen.

p) Kidney Biopsy: Diffuse Lupus Nephritis Class IV of ISN/RPS classification with active lesion.

We diagnosed our patient as Bullous systemic lupus erythematosus (BSLE) with ISN/RPS class IV lupus nephritis with active lesion presenting with rapidly progressive glomerulonephritis (RPGN).

Initially patient was managed with Methylprednisolone pulse therapy 1gm once a day IV for three days followed by oral Prednisolone with Hydroxychloroquine and systemic broad spectrum antibiotics from day one. Dapsone was started with $100 \mathrm{mg}$ BD for bullous lesion but she was intolerant to Dapsone. Eventually her renal biopsy report came on day $10^{\text {th }}$ of admission; with suggestion of active lupus nephritis, she was put on Rituximab $375 \mathrm{mg} / \mathrm{square}$ meter body surface area.

Later we received her skin biopsy report only to corroborate our clinical impression and forming our final diagnosis.

One month after completion of two doses of Rituximab, her renal impairment and skin lesions improved significantly. Biochemical assessment showed normalisation of creatinine value $(1.2 \mathrm{mg} / \mathrm{dl})$ with a creatinine clearance of $60 \mathrm{ml} / \mathrm{min}$ and reduction of proteinuria. In her subsequent visit after six months of $4^{\text {th }}$ dose of Rituximab, proteinuria reduced further and renal recovery was nearly complete with creatinine clearance of $74 \mathrm{ml} / \mathrm{min}$ without any further complication.

\section{Discussion}

BSLE accounts for less than one per cent of skin manifestations, ${ }^{1}$ generally affects young adult females and involves both sun-exposed and non-exposed areas. Because of the particularly clinical and histological presentation of BSLE, Camisa and Sharma proposed diagnostic criteria for BSLE; these include i) A diagnosis of SLE based on the ACR criteria; ii) Vesicles and bullae mainly located on sunexposed areas; iii) The histopathology is characterised by sub-epidermal bullae with micro abscesses of neutrophils in the dermal papillae, similar to those found in dermatitis 
herpetiformis; and iv) Deposition of IgG, IgM, or both and often IgA in the basement membrane zone. ${ }^{2}$

Rituximab which is a chimeric monoclonal antibody that reacts with $C D 20$, an antigen that is present on immature, naive, and memory $B$ cells but not on mature plasma cells, has been approved in the treatment of SLE with good efficacy and safety. ${ }^{3}$

In this case report we have used Rituximab as an immuno modulator in a complicated life threatening situation of target organ damage. We have experienced a dramatic response in terms of skin lesions and renal recovery in our patient. Hydroxychloroquine and tapering dose of Prednisolone was prescribed to his patient and even after three successive follow up one month and six months apart no more bullous lesion was observed in this patient and her renal recovery was satisfactory.

Till now, there is only one case report showing improvement of skin lesion with Rituximab as a treatment of BSLE, but unlike this case Mycophenolate mofetil was used alongside. ${ }^{4}$ In this case a satisfactory response solely due to Rituximab has been observed even in an advanced life threatening nephropathy situation. This is obvious that potential role of Rituximab as an immuno modulator cannot be left aside. ${ }^{5}$

\section{Conclusion}

Rituximab can be given as an alternative to Dapsone in bullous lesion associated with SLE and as an immuno modulator in active lupus nephritis with satisfactory renal outcome.

\section{References}

1. Gammon WR, Briggaman RA. Bullous eruption of systemic lupus erythematosus. In: Wojnarowska $F$, Briggaman RA, editors. Management of Blistering Diseases. London: Chapman and Hall Ltd; 1990;263-75.

2. Camisa C, Sharma HM. Vesiculobullous systemic lupus erythematosus. Report of two cases and a review of the literature. J Am Acad Dermatol. 1983;9(6):924-933.

3. Chanprapaph K, Sawatwarakul S, Vachiramon V. A 12year retrospective review of bullous systemic lupus erythematosus in cutaneous and systemic lupus erythematosus patients. Lupus. 2017;26:1278-1284.

4. Cobo-Ibáñez T, Loza-Santamaría E, Pego-Reigosa JM, et al. Efficacy and safety of rituximab in the treatment of non-renal systemic lupus erythematosus: a systematic review. Semin Arthritis Rheum. 2014;44(2):175-185.
5. Alsanafi S, Kovarik C, Mermelstein AL, et al. Rituximab in the treatment of bullous systemic lupus erythematosus. J Clin Rheumatol. 2011;17(3):142-144.

\section{ACKNOWLEDGEMENTS}

Director IPGME\&R Kolkata.

Dean of Students affairs IPGME\&R Kolkata.

\section{PEER REVIEW}

Not commissioned. Externally peer reviewed.

\section{CONFLICTS OF INTEREST}

The authors declare that they have no competing interests.

\section{FUNDING}

None

\section{PATIENT CONSENT}

The authors, Chakraborty A, Ghosh S, Ghosh S, Chowdhury $A$, Mondal $D$, declare that:

1. They have obtained written, informed consent for the publication of the details relating to the patient(s) in this report.

2. All possible steps have been taken to safeguard the identity of the patient(s).

3. This submission is compliant with the requirements of local research ethics committees.

Figure 1: Bullous lesion with secondary infection in neck

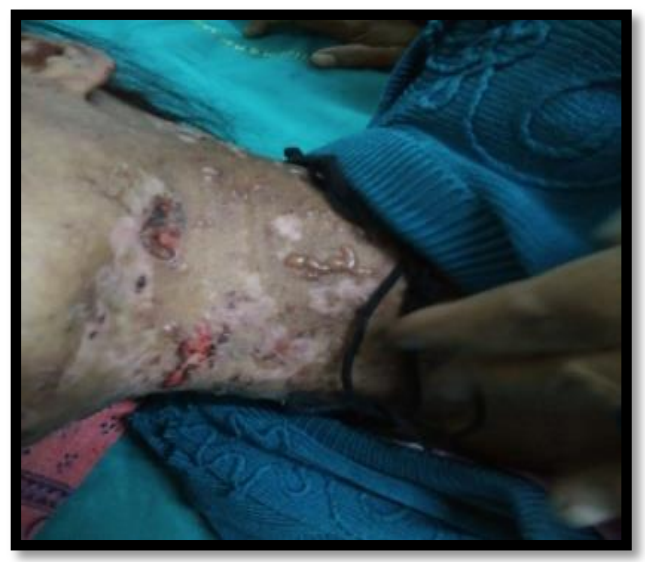


Figure 2: Bullous lesion with inflammatory hyper and hypo pigmentation in face

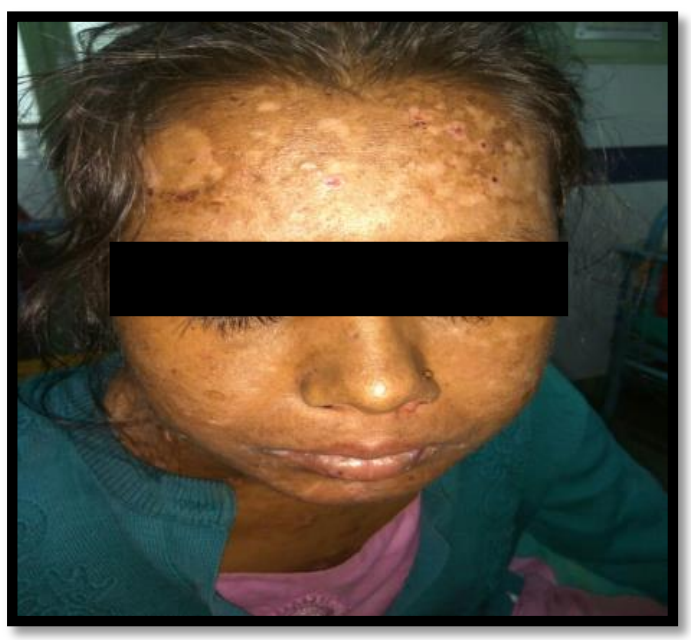

Figure 3: Vesicobullous lesion

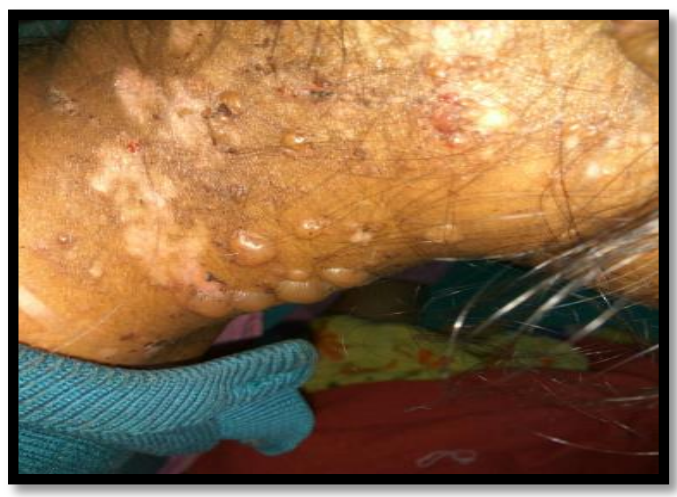

Figure 4: Skin biopsy showing Showing Bullous lesion with neutrophilic infiltrate and sub epidermal separation

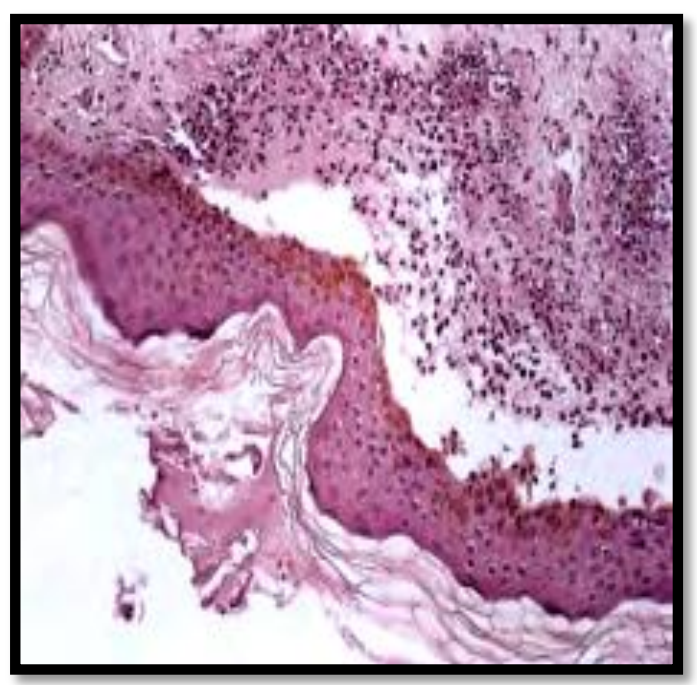

Figure 5: Index patient after recovery

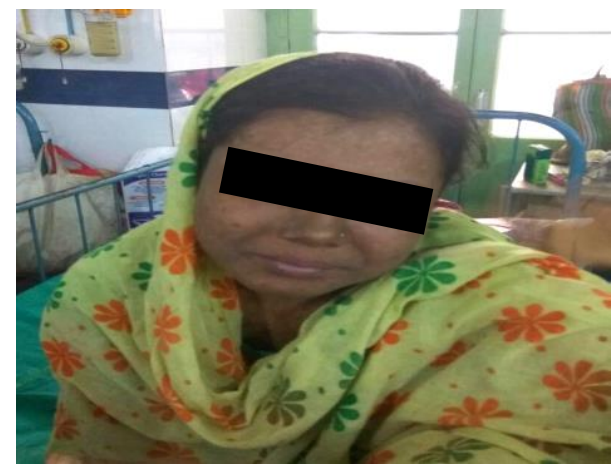

Table 1: Blood Biochemistry and Complete blood counts

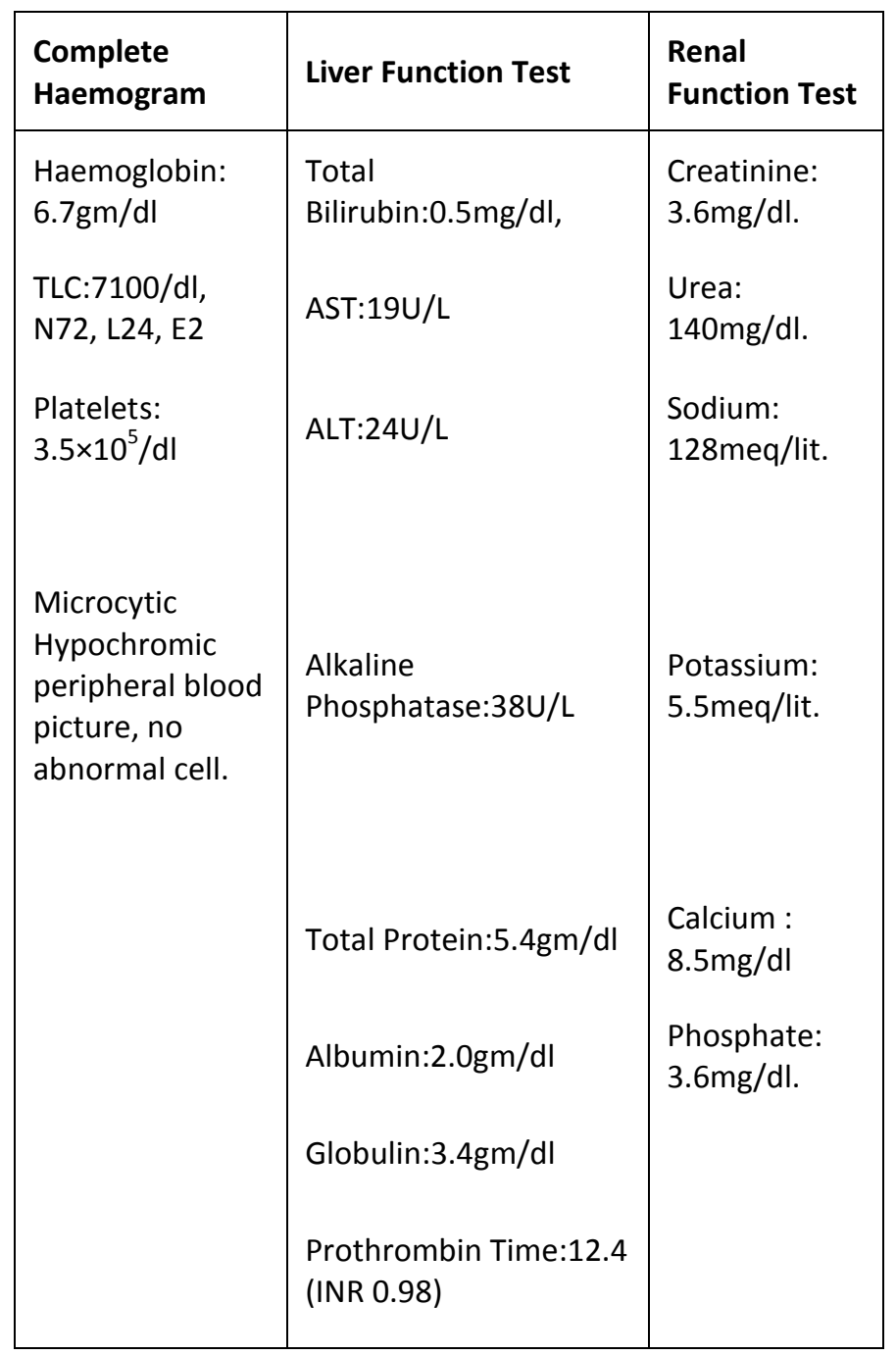

